\title{
Preconditioning donor with a combination of tacrolimus and rapamacyn to decrease ischaemia-reperfusion injury in a rat syngenic kidney transplantation model
}

F. Cicora, ${ }^{\star \star}{ }^{\star}$ J. Roberti, ${ }^{\ddagger}$ D. Vasquez,,

D. Guerrieri, ${ }^{\dagger}$ N. Lausada, ${ }^{\star}$ P. Cicora ${ }^{\dagger}$ G. Palti, ${ }^{\S}$ E. Chuluyan, ${ }^{`}$ P. Gonzalez, ${ }^{\star *}$ P. Stringa ${ }^{\star}$ and C. Raimondi ${ }^{\star}$ ${ }^{*}$ Organs and Tissue Transplant Program of The Faculty of Medicine, Universidad de la Plata, ${ }^{\dagger}$ Foundation for the Research and Treatment of Kidney Disease - Fundación para la Investigación y Asistencia de la Enfermedad Renal (FINAER), La Plata, ${ }^{\ddagger}$ Universidad de Belgrano, ${ }^{\lessgtr}$ Kidney Transplant Unit, Hospital Alemán, Ciudad de Buenos Aires, "Pharmacology Department of the Faculty of Medicine, Universidad de Buenos Aires and ${ }^{* *}$ Comisión de Investigaciones Científicas (CIC), Buenos Aires, Argentina

Accepted for publication 15 September 2011 Correspondence: F. Cicora, Unidad de Trasplante Renal, Hospital Alemán, Pueyrredón 1640, C1118AAT Ciudad de Buenos Aires,

Argentina.

E-mail: fcicora5@gmail.com

\section{Summary}

Reperfusion injury remains one of the major problems in transplantation. Repair from ischaemic acute renal failure (ARF) involves stimulation of tubular epithelial cell proliferation. The aim of this exploratory study was to evaluate the effects of preconditioning donor animals with rapamycin and tacrolimus to prevent ischaemia-reperfusion (I/R) injury. Twelve hours before nephrectomy, the donor animals received immunosuppressive drugs. The animals were divided into four groups, as follows: group 1 control: no treatment; group 2: rapamycin $(2 \mathrm{mg} / \mathrm{kg})$; group 3 FK506 $(0,3 \mathrm{mg} / \mathrm{kg})$; and group 4: FK506 (0, $3 \mathrm{mg} / \mathrm{kg})$ plus rapamycin $(2 \mathrm{mg} / \mathrm{kg})$. The left kidney was removed and after $3 \mathrm{~h}$ of cold ischaemia, the graft was transplanted. Twentyfour hours after transplant, the kidney was recovered for histological analysis and cytokine expression. Preconditioning treatment with rapamycin or tacrolimus significantly reduced blood urea nitrogen and creatinine compared with control [blood urea nitrogen (BUN): $P<0.001$ versus control and creatinine: $P<0.001$ versus control]. A further decrease was observed when rapamycin was combined with tacrolimus. Acute tubular necrosis was decreased significantly in donors treated with immunosuppressants compared with the control group $(P<0.001$ versus control). Moreover, the number of apoptotic nuclei in the control group was higher compared with the treated groups $(P<0.001$ versus control). Surprisingly, only rapamycin preconditioning treatment increased anti-apoptotic $\mathrm{Bcl} 2$ levels $(P<0 \cdot 001)$. Finally, inflammatory cytokines, such as tumour necrosis factor (TNF)- $\alpha$ and interleukin (IL)-6, showed lower levels in the graft of those animals that had been pretreated with rapamycin or tacrolimus. This exploratory study demonstrates that preconditioning donor animals with rapamycin or tacrolimus improves clinical outcomes and reduce necrosis and apoptosis in kidney I/R injury.

Keywords: acute tubular necrosis, apoptosis, donor preconditioning, rapamycin, tacrolimus

\section{Introduction}

Ischaemia-reperfusion injury (I/R injury), the most important non-immunological determinant of kidney injury, is still one of the major problems in kidney transplantation. I/R injury can increase acute rejection rate and decrease longterm allograft survival. I/R injury in the kidney is expressed as acute renal dysfunction, evidenced by acute tubular necrosis and apoptosis $[1,2]$. The deleterious effects of I/R injury are triggered by a complex response involving damageassociated molecular pattern molecules (DAMPs), oxygen radical species, cytokines, chemokines and complement
$[3,4]$. These inflammatory events induce apoptosis and necrosis in renal cells, initiated through either the mitochondrial pathway or the receptor-mediated pathway, such as binding of tumour necrosis factor (TNF- $\alpha$ ) to their corresponding receptors [5]. During the past few years, it has been documented that cell apoptosis in I/R injury is also associated with complement activation [6,7]. Both anaphylotoxin (C3a, C5a) and I/R injury membrane attack complex mechanisms have been proposed as means by which the complement cascade induces tissue injury in an animal model of renal I/R injury $[8,9]$. Furthermore, the use of an anti-C5 antibody has been shown to prevent the development of 
apoptosis after renal and cardiac I/R injury [10]. I/R injury is an antigen-independent inflammatory process that produces tissue damage [11].

There are different strategies to choose from and different potential intervention aspects of the natural development of the disease. We could potentially modify factors related to donors, preservation solutions and recipients. Treating the donor with different drugs is among the new strategies to improve the quality of procured organs in renal transplant; for example, steroids and statins [12-14].

Rapamycin, an antibiotic that inhibits protein synthesis through mammalian target of rapamycin (mTOR) signalling, has been used to attenuate I/R injury immediately posttransplant without promising results [15]. Tacrolimus, an antibiotic that inhibits calcineurin, administered to donors has been reported to attenuate I/R injury [16]. Following our previous studies [17], in which a kidney autotransplant model was used, we observed that rapamycin treatment was more effective in the prevention of apoptosis, whereas treatment with tacrolimus presented the lowest levels of acute tubular necrosis (ATN), so we explored the synergic effects of both drugs, rapamycin and tacrolimus, when they were administered to the donor.

Although the effects of rapamycin on lymphocytes have been investigated thoroughly, it is known that this drug also has a direct effect on renal proximal tubular cell function [18]. Repair from ischaemic acute renal failure involves stimulation of tubular epithelial cell proliferation. Agents impairing the ability of renal epithelium to proliferate, especially in the face of ongoing injury, may result in prolonged periods of acute renal failure (ARF) or failure in recovery. Several studies of ARF have shown augmented injury and delay repair when rapamycin is given near the time of injury $[19,20]$. The mechanism appears to involve a combination of enhanced necrosis, increased apoptosis and decreased proliferation of renal tubular epithelial cells. In contrast, it has been demonstrated that treatment with rapamycin in the recipient animals attenuated I/R injury in small bowel [21] and kidney I/R injury [22,23]. Also it has been reported that rapamycin has a potent preconditioning effect in an animal model of heart I/R injury [24]. However, it is well known that rapamycin could aggravate ischaemically injured organs, increasing cell apoptosis and negatively affecting posttransplantation recovery $[15,20]$. Conversely, tacrolimus is a calcineurin inhibitor normally administered to receptors of renal transplant to block the activation of nuclear factor of activated T cells (NF-AT) [25]. Tacrolimus produces multifaceted attenuating actions on inflammatory damage occurring after reperfusion. Lastly, pretreatment with tacrolimus has been shown to provide liver and renal protection against $\mathrm{I} / \mathrm{R}$ injury in rats $[26,27]$.

Although intervention in the preservation solution and the receptor has always been the first choice, because of insufficient evidence supporting a successful intervention in the donor there has always been research into the adminis- tration of immunosuppressive drugs to the donor. Before transplantation, the kidney already contains several infiltrated macrophages and T lymphocytes [28]. This inflammatory process, activated by cold ischaemia as well as brain death, may be explained by changes in the kidney tissue itself [29]. Another potential reason is that these inflammatory mediators could be released from $\mathrm{T}$ lymphocytes and macrophages infiltrated in the kidney. Therefore, the administration of rapamycin and tacrolimus to the donor could be useful to inhibit the release of mediators from the graft [30]. Anticipating the inflammatory process through the administration of immunosuppressive drugs to the donor could be one of the scenarios to reduce the graft immunogenicity.

In previous studies, we have used tacrolimus and rapamycin separately, and we observed a reduction in the in-situ generation of proinflammatory mediators and an up-regulation of cytoprotective genes [17]. We hypothesized that the combined use of rapamycin and tacrolimus treatment in donor animals would be associated with the attenuation of $\mathrm{I} / \mathrm{R}$ injury. In addition, it has been observed that rapamycin had a tendency to decrease apoptosis and that tacrolimus had a tendency to decrease ATN. Therefore, we have hypothesized that the combination of these two drugs in donor animals could have a synergetic effect to decrease necrosis and apoptosis.

\section{Materials and methods}

\section{Animals}

Male Wistar rats weighing 280-350 g were used for both organ donors and recipients of the kidney graft. Animals were submitted to a 12-h day/night cycle with access to water and standard laboratory chow ad libitum. All animal experiments were performed according to guidelines set by the US National Institutes of Health (NIH publication no. 28, revised 1996).

\section{Immunosuppressive drugs}

We used tacrolimus (Prograf, Gador, Bs As, Argentina), medical grade, donated by Gador Argentina, and rapamycin (Sirolimus, Wyeth, Bs As, Argentina), medical grade, donated by Wyeth Argentina.

\section{Operation procedure}

Donor rats were anaesthetized with intraperitoneal (i.p.) atropine $0.01 \mathrm{mg} / \mathrm{kg}$, buprenorphine $0.04 \mathrm{mg} / \mathrm{kg}$, diazepam $10 \mathrm{mg} / \mathrm{kg}$ and, $10 \mathrm{~min}$ later, with ketamine $100 \mathrm{mg} / \mathrm{kg}$ body weight. The donors' blood vessels and ureter were fully separated. Subsequently, the kidney was flushed via the aorta with $3 \mathrm{ml}$ of $4^{\circ} \mathrm{C}$ cold Ringer lactate solution until it turned homogeneously pale. The left kidney was then removed with its vascular and ureteral pedicle and stored for $180 \pm 15 \mathrm{~min}$ in cold Ringer lactate solution at $4^{\circ} \mathrm{C}$. 
Recipients animals were nephrectomized bilaterally and underwent transplantation as described elsewhere [31,32]. Briefly, after flushing grafts with $5 \mathrm{ml}$ normal Ringer's solution, arterial and venous anastomoses were performed as end-to-side anastomoses to the aorta and inferior vena cava, respectively. Finally, the anastomosis of the ureter with the urinary bladder was constructed. The rat's body temperature was monitored and kept constant between 35 and $37^{\circ} \mathrm{C}$ in all cases. Rats were allowed to recover on a warm blanket with free access to water and standard laboratory chow ad libitum. Twenty-four hours after the transplant procedure, blood samples were obtained for analysis, then animals were sacrified and kidneys were removed for histological evaluation.

\section{Experimental design}

One dose of immunosuppressive drugs was administered to donor animals $12 \mathrm{~h}$ before nephrectomy. Doses and administration route were chosen according to previous reports [17]. Donor rats were divided randomly into four groups:

Group 1 (control, $n=6$ ): no immunosuppression was administered.

Group 2 (rapa, $n=6$ ): rapamycin $(2 \mathrm{mg} / \mathrm{kg}$, Sirolimus, Wyeth, Argentina) by gavage.

Group 3 (FK506, $n=6$ ): tacrolimus $(0,3 \mathrm{mg} / \mathrm{kg}$, Prograf, Gador, Argentina) by gavage.

Group 4 (rapa+ FK506, $n=6$ ): tacrolimus $(0,3 \mathrm{mg} / \mathrm{kg})+$ rapamycin $(2 \mathrm{mg} / \mathrm{kg})$ by gavage.

None of the recipient animals received any immunosuppressive drug after transplantation. In addition, six rats underwent a sham procedure.

\section{Blood tests}

Twenty-four hours before and after transplant the following blood determinations were performed: blood urea nitrogen (BUN), creatinine and C3 complement fraction (C3). C3 was measured by radial immunodiffusion and BUN and creatinine by ultraviolet kinetic and colorimetric-kinetic, respectively (Mindray 300). Values are expressed in figures as the difference between post-transplant and pretransplant were defined for each group.

\section{Renal histopathology}

The anatomopathological samples were analysed by a pathologist blind to group assignments. The kidneys were fixed in a $10 \%$ neutral buffered formalin solution, embedded in paraffin and used for histopathological examination. Four micrometres-thick sections were cut, deparaffinized, hydrated and stained with haematoxylin and eosin (H\&E). The renal sections were examined in a blinded fashion for grade of cortical tubular epithelial necrosis. Counts were performed in at least 10 different fields of square micrometres and assigned for severity of necrosis, using scores on a scale of $1(<5 \%), 2(5-25 \%), 3(25-50 \%), 4(50-75 \%)$ and $5(>75 \%)$ [23].

\section{Terminal transferase deoxuridine triphosphate (dUTP) nick end labelling (TUNEL) assay}

TUNEL assay was performed according to the manufacturer's instructions (Apoptag; Oncor, Gaithersburg, MD, USA). Briefly, deparaffinized $4 \mu \mathrm{m}$-thick sections of paraffinembedded tissues were pretreated with $20 \mu \mathrm{l} / \mathrm{ml}$ Proteinase $\mathrm{K}$ (Dako, Glostrup, Denmark) for $30 \mathrm{~min}$ at $37^{\circ} \mathrm{C}$. After washing, sections were incubated with digoxygenin-labelled dUTP in the presence of terminal deoxynucleotidyl transferase. After the enzymatic reaction was blocked, sections were incubated with a specific peroxidase-labelled antidigoxin antibody. Peroxidase was then reduced by 0.05 diaminobenzidine (Sigma, St Louis, MO, USA) in $0 \cdot 1 \mathrm{ml} / \mathrm{l}$ phosphate-buffered saline (PBS), $\mathrm{pH} 7 \cdot 6$ containing $1 \%$ $\mathrm{H}_{2} \mathrm{O}_{2}$. After washing, the sections were lightly stained with haematoxylin. Negative control reactions were performed for each reaction step. They were obtained by omission of terminal deoxynucleotidyl transferase, anti-digoxin antibody and peroxidase substrate. Positive controls included sections of paraffin-embedded lymphoma of human origin. The external medullar region was examined and the total number of labelled nuclei was counted. Ten fields of $1 \mathrm{~mm}^{2}$ were examined by means of a reticulated lens.

\section{Immunohistochemistry}

Sections $4 \mu \mathrm{m}$ thick were applied to poly-2-lysine coated slides. Sections were dewaxed in xylene, dehydrated through graded alcohols and water and then immersed in $0 \cdot 3 \% \mathrm{vol} / \mathrm{vol}$ $\mathrm{H}_{2} \mathrm{O}_{2}$ in methanol for $30 \mathrm{~min}$ to block endogenous peroxidase. Antigens were reduced by microwaving at $750 \mathrm{~W}$ for $15 \mathrm{~min}$ in $0.01 \mathrm{~mol} / \mathrm{l}$ trisodium citrate buffer, $\mathrm{pH} 6 \cdot 0$, then rinsed well in standard PBS and non-specific binding was blocked with $10 \%$ equine serum in PBS. Sections were incubated with primary antibodies of monoclonal origin against C3 (clone B-9) or with polyclonal from goat against TNF- $\alpha$, interleukin (IL)-6 and Bcl2 (Santa Cruz Biotechnology, Santa Cruz, CA, USA). After being rinsed with PBS, sections were incubated with biotinylated secondary antibodies. Afterwards, sections were rinsed with PBS and incubated with avidin-biotin horseradish peroxidase complex according to the manufacturer's instructions (Vectastain Universal Quick Kits; Vector Laboratories Ltd, Peterborough, UK). Peroxides were visualized by incubating the sections in $3 \cdot 3^{\prime}$ diaminobenzidine (Sigma Chemical Co., Poole, UK) and hydrogen peroxide. Negative control experiments were performed by omitting the incubation with the primary antibodies. The presence of C3, TNF- $\alpha$, IL- 6 and Bcl 2 was assessed in 10 consecutive cortex and medulla fields. 
Immunohistochemical area and optical density determination

Images were captured from a microscope (Olympus BX50, Tokyo, Japan) with a $\times 4$ objective through an attached digital video camera (Olympus DP71, Tokyo, Japan) as TIF, RGB images. The entire section was scanned with the help of a motorized stage (Prior Scientific Inc., Rockland, MA, USA). Stitched images were then analysed using image analysis software (ImagePro Plus 6.3; Media Cybernetics Inc, Bethesda, MD, USA). The entire section area of the slice was calculated. To separate the positive immunostaining area (brown stain) from the background, the colour segmentation function of the program was applied. A mask was then applied to make the colour separation permanent. The images were then transformed into 8-bit monochromatic. After spatial and intensity of light calibration of the images, the stained area and its optical density (OD), defined by the antigen-antibody complex, were determined [33]. The extension and the intensity of these markers was evaluated and an immunohistochemical score (IS) was generated; IS $=($ stained area/total area $) \times$ intensity.

\section{Statistical analysis}

All values are expressed as mean \pm standard deviation of the mean (s.d.). Analysis of variance (ANOVA) was used to determine group differences. If the ANOva was significant, multiple comparisons were carried out using the Bonferroni post-hoc test to locate the sources of differences. Nonparametric variables were analysed with the Kruskal-Wallis non-parametric ANOvA. $P<0.05$ was considered to indicate a statistically significant difference.

\section{Results}

\section{Plasma determination of BUN and creatinine}

Plasma determinations were measured $24 \mathrm{~h}$ after transplant procedure. Compared with the control group, BUN values in the immunosuppressive treatment groups were significantly reduced (BUN: control: $2 \cdot 2 \pm 0 \cdot 15 \mathrm{mg} / \mathrm{dl}$; rapamycin $1.8 \pm 0.15 \mathrm{mg} / \mathrm{dl} ;$ FK506 $1.6 \pm 0.15 \mathrm{mg} / \mathrm{dl} ;$ rapamycin + FK506 $1 \cdot 3 \pm 0 \cdot 1 \mathrm{mg} / \mathrm{dl} ; P<0 \cdot 001$ versus control) (Fig. 1a). In the rapamycin + FK506 group, BUN values were significantly lower than those in rapamycin or FK506 single treatment $(P<0.001, P<0.05$, respectively). Among single treatments, BUN level was lower in FK506 than with rapamycin $(P<0 \cdot 01)$. In the case of creatinine, compared with control values, the immunosuppressive treatment groups were reduced significantly (control: $4.7 \pm 1.34 \mathrm{mg} / \mathrm{dl}$; rapamycin $2 \cdot 1 \pm 0 \cdot 1 \mathrm{mg} / \mathrm{dl} ;$ FK506 $2 \pm 0.31 \mathrm{mg} / \mathrm{dl}$; rapamycin + FK506 1.1 $\pm 0.13 \mathrm{mg} / \mathrm{dl} ; P<0 \cdot 001$ versus control) (Fig. $1 \mathrm{~b}$ ). However, no variances were observed between the differ- (a)

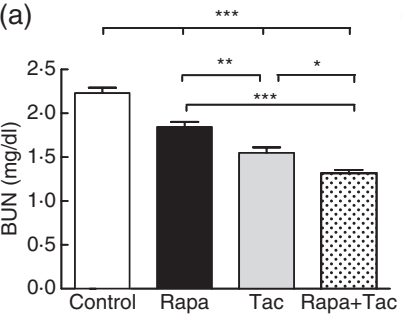

(b)

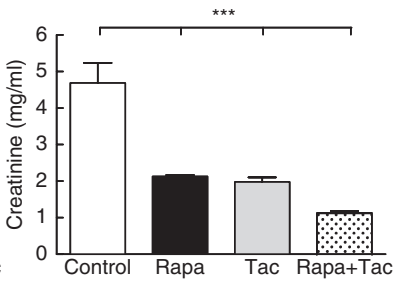

Fig. 1. Plasma blood urea nitrogen (BUN) and creatinine after transplantation. Twenty-four hours after transplantation blood samples were obtained and serum parameters (a) BUN and (b) creatinine were determined. Values are expressed as the difference between post-transplantation minus pretransplantation for each group. Values are mean \pm standard deviation $(n=6) .{ }^{\star} P<0 \cdot 05$, ${ }^{* *} P<0.01$ and ${ }^{* * *} P<0.001$ for comparison between groups. No differences among sham animals were observed.

ent immunosuppressive treatments over creatinine levels $(P>0 \cdot 05)$. In the sham group, there were no differences in urea and plasma creatinine between pre- and post-surgical procedures (BUN pre-: 0.43 $\pm 0.01 \mathrm{mg} / \mathrm{dl}$ and post-: $0.43 \pm$ $0.03 \mathrm{mg} / \mathrm{dl} P>0.05$; creatinine pre-: $0.88 \pm 0.06 \mathrm{mg} / \mathrm{dl}$ and post-: $0 \cdot 89 \pm 0.05 P>0 \cdot 05)$. These results in BUN and creatinine suggest that immunosuppressive treatment applied to the donor reduced post-transplant renal dysfunction significantly.

\section{Kidney damage}

Necrosis and kidney damage were assessed with H\&E-stained kidney tissue $24 \mathrm{~h}$ after transplantation. Acute tubular necrosis score (ATN) was decreased significantly in the immunosuppressive treatment group compared with the control group ( $4 \pm 0.63$ in control; rapamycin $2 \cdot 2 \pm 0 \cdot 41$; FK506 $2 \pm 0.63$; rapamycin + FK506 $1 \cdot 2 \pm 0 \cdot 41 ; P<0.001$ versus control; Fig. 2a). Figure $2 \mathrm{~b}$ shows a representative image of $\mathrm{H} \& \mathrm{E}$ stain for the evaluation of renal injury in each treatment group. The use of rapamycin plus tacrolimus (group 4) was associated with a lower level of acute tubular necrosis (ATN) compared with rapamycin alone $(P<0 \cdot 05)$, but no statistical difference was observed in comparison with tacrolimus. Also, the number of apoptotic nuclei in renal medulla was determined as evidence of kidney injury. In the control group, the number of TUNEL-positive cells was higher compared with the immunosuppressive treatment groups (control: $138 \cdot 7 \pm 24 \cdot 8$; rapamycin: $22 \cdot 3 \pm 4 \cdot 5$; FK506: $54 \cdot 8 \pm 8 \cdot 3$ and rapamycin + FK506: $17 \cdot 5 \pm 5 ; \quad P<0.001$ versus control, Fig. $3 \mathrm{a}$ and b). As normal kidney control, the number of positive apoptotic nuclei in sham animals was lower than $6 / \mathrm{mm}^{2}$ located only in deep medullary epithelial tubules (data not shown). The use of rapamycin alone or rapamycin plus tacrolimus showed a lower number of apoptotic nuclei cells with respect to tacrolimus treatment $(P<0.05$ and $P<0.01$, respectively). Finally, a statistically significant difference in the expression of $\mathrm{Bcl} 2$ was detected in kidney tissue by 
(a)

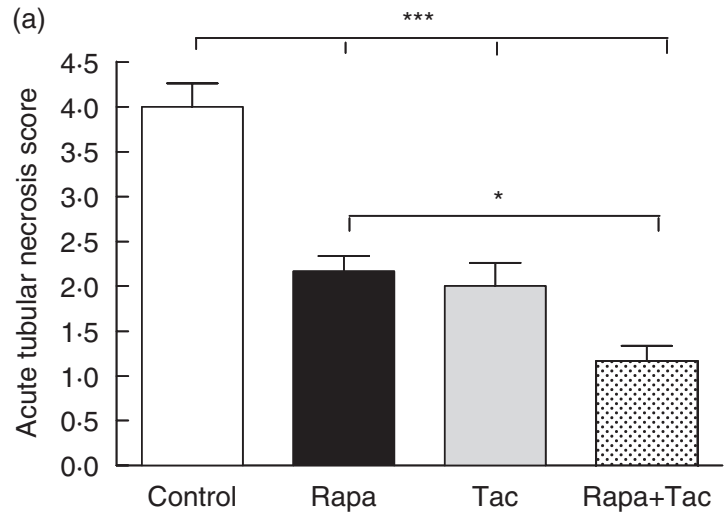

(b)
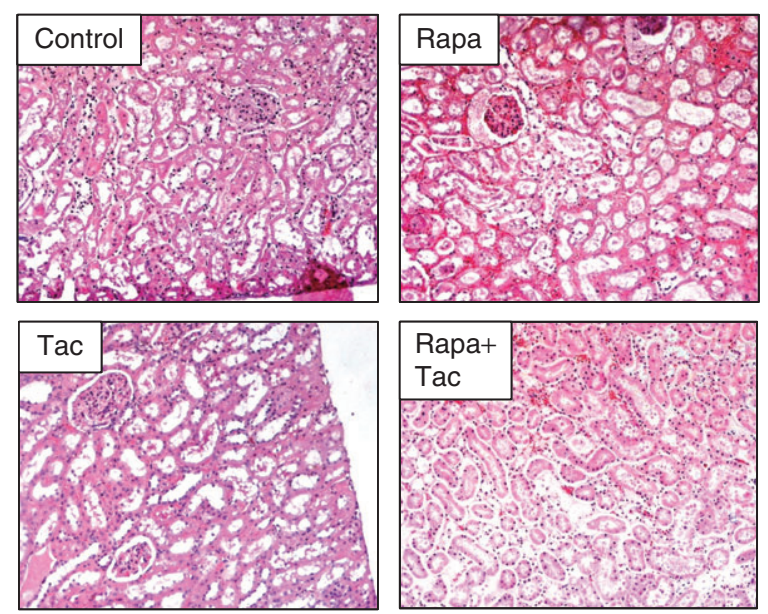

Fig. 2. Kidney tubular damage after transplantation. (a) The acute tubular necrosis score was graded in haematoxylin and eosin (H\&E) staining cortex according to particular histological findings from 1 to 5 . Values are mean \pm standard deviation $(n=6) .{ }^{*} P<0.05$ and ${ }^{* * *} P<0.001$ for comparison between groups. (b) Representative H\&E stain for the evaluation of renal injury after $24 \mathrm{~h}$ of ischaemia-reperfusion.

immunohistochemistry. In accordance with our previous results, Bcl2 levels in the control group were lower than in the immunosuppressive treatment group (control: $1 \cdot 8 \pm 0 \cdot 5$; rapamycin: 16.01 \pm 4 ; FK506: $9 \pm 2.6$ and rapamycin + FK506: $6 \pm 1.25 ; P<0.01$ and $P<0.05$ versus control, respectively) (Fig. $3 \mathrm{c}$ ). These results suggest that preconditioning of the donor with rapamycin and tacrolimus or a combination of both is associated with lower kidney damage after transplantation.

\section{Complement C3 determination}

In order to determine if the immunosuppressive treatment affected the complement function, the $\mathrm{C} 3$ levels in recipient animals were assessed. C3 plasma values in immunosuppressive treatment were significantly lower than control group levels (control: $495 \pm 94 \mathrm{pg} / \mathrm{ml}$; rapamycin: $166 \cdot 7 \pm$ $57 \cdot 1 \mathrm{pg} / \mathrm{ml}$; FK506: $165 \pm 66 \cdot 3 \mathrm{pg} / \mathrm{ml}$ and rapamycin + FK506: $103.3 \pm 33.3 . ; P<0.001$ versus control, Fig. $4 \mathrm{a})$. No differences were found among the various immunosuppressive treatment groups $(P>0 \cdot 05)$. In addition, the local expression of C3 within the grafts was analysed. Immunohistochemical analysis of graft tissue $24 \mathrm{~h}$ after transplantation revealed that local expression of $\mathrm{C} 3$ was higher in the control group compared with the immunosuppressive treatment group (control: $53.98 \pm 4.5$; rapamycin: $10 \cdot 62 \pm 3 \cdot 2$; FK506: $2.27 \pm 0.7$ and rapamycin + FK506: $1.58 \pm 0.54$; $P<0.001$ versus control; Fig. $4 \mathrm{~b}$ and $\mathrm{c})$. There were no variances among the different drug treatments used $(P>0 \cdot 05)$.

\section{Cytokine expression}

Finally, local expression of TNF- $\alpha$ and IL- 6 was analysed by immunohistochemistry in kidney tissue $24 \mathrm{~h}$ after transplantation. Higher levels of TNF- $\alpha$ were observed (control: 57.54 \pm 5.7; rapamycin: $2 \cdot 7 \pm 0 \cdot 99 ;$ FK506: $2 \cdot 83 \pm$ 1.02 and rapamycin + FK506: $4.43 \pm 1.5 ; P<0.001$ versus control) and IL-6 in the control group compared with immunosuppressive treatment groups (control: $30 \cdot 43 \pm 4 \cdot 6$; rapamycin: $2 \cdot 31 \pm 2 \cdot 05$; FK506: $3.73 \pm 3.6$ and rapamycin + FK506: $6.57 \pm 2 \cdot 8 ; P<0.001$ versus control, Fig. 5$)$. There was no variance between the treatment groups $(P>0 \cdot 05)$.

\section{Discussion}

This study suggests that a single dose of a combination of rapamycin and tacrolimus given to donors could attenuate the I/R injury caused by cold ischaemia. There appears to be a clinical and histological improvement and reduction of inflammatory mediators without administration of drugs in the recipient after transplantation. To the best of our knowledge, this is the first report to use an isogenic transplant model to study the effects of combined preconditioning treatment with rapamycin and tacrolimus in donors for renal I/R injury.

Our findings are in line with previous studies demonstrating that preconditioning donors with calcineurin inhibitors (CNI) can protect the kidney from I/R injury [16,34]. However, the basic mechanism behind CNI preconditioning remains unknown. In our model, $24 \mathrm{~h}$ after the I/R injury process, the presence of acute renal failure was expressed clinically by plasmatic urea and creatinine increases and expressed histopathologically by necrosis and apoptosis. Preconditioning with immunosuppressive drugs applied to the donor attenuated renal dysfunction, as BUN and plasma $\mathrm{Cr}$ levels were reduced significantly with the immunosuppressive treatment. The combined therapy with rapamycin and tacrolimus generated lower levels of BUN and creatinine. These results are in contrast with previous reports showing that rapamycin alone or in combination with tacrolimus delays recovery $\mathrm{I} / \mathrm{R}$ injury in warm ischaemic models $[35,36]$. We hypothesized that this dual effect of rapamycin, depending on the time of administration, could be the reason why an improvement in graft function was observed. 
(a)

Fig. 3. Apoptosis and $\mathrm{Bcl} 2$ expression. (a) Apoptotic cells using the terminal deoxynucleotidyl transferase deoxuridine triphosphate (dUTP) nick end labelling (TUNEL) method in outer and inner medulla. The total number of labelled nuclei was counted in 10 fields of $1 \mathrm{~mm}^{2}$. As normal kidney control, the number of positive apoptotic nuclei in sham animals was lower than $6 / \mathrm{mm}^{2}$ located only in deep medullary epithelial tubules. (b) Representative TUNEL staining of kidney sample after $24 \mathrm{~h}$ of reperfusion. Positive apoptotic cells appear with brown stained nuclei in outer medulla and cortex $(A \times 100$, scale bar $=100 \mu)$. (c) Immunohistochemical score of Bcl2 in renal tissue from transplanted rats. Quantitative analysis of $\mathrm{Bcl} 2$ expression was assessed in 10 consecutive cortex and medulla fields. Values are mean \pm standard deviation $(n=6)$. ${ }^{*} P<0.05,{ }^{*} P<0.01$ and ${ }^{* *} P<0.001$ for comparison between groups.

It should be noted that these studies were performed with models of warm ischaemia and that immunosuppressants were administered before and after the induction of $I / R$ injury. In our work, we used a model of cold ischaemia with administration of immunosuppression to the donor only before transplantation.

We cannot ignore that the effect of different immunosuppressants on $\mathrm{I} / \mathrm{R}$ injury after renal transplantation is not always clear. For example, cyclosporin has shown to impair the recovery of renal allograft from delayed graft function (DGF) [37]. In the case of rapamycin, Inman et al. have demonstrated that rapamycin preserves function compared with cyclosporin after I/R injury [22]. In contrast, several authors have demonstrated that rapamycin treatment in I/R injury was associated with elevated serum creatinine levels, reduced proliferative response of renal tubular cells and increased expression of transforming growth factor (TGF)- $\beta$ [38], leading to impaired renal recovery after I/R injury $[20,23]$. In the immunostimulation setting after transplant, rapamycin decreases lymphocyte proliferation and reduces rejection [39]. Nevertheless, in the setting of renal injury, where organ repair depends on tubular cell proliferation and well-orchestrated apoptosis, rapamycin may be harmful. Lieberthal et al. [40] have demonstrated that rapamycin inhibits proliferation and increases apoptosis of renal tubular epithelial cells in vitro and in vivo. Furthermore, there is evidence of pharmacokinetic interactions between rapamycin and CNI that augment ischaemic injury and inhibit tissue repair when used in combination [41]. Conversely, our results may demonstrate that the combination of rapamycin and tacrolimus administered to donors decreases apoptosis and necrosis in (b)
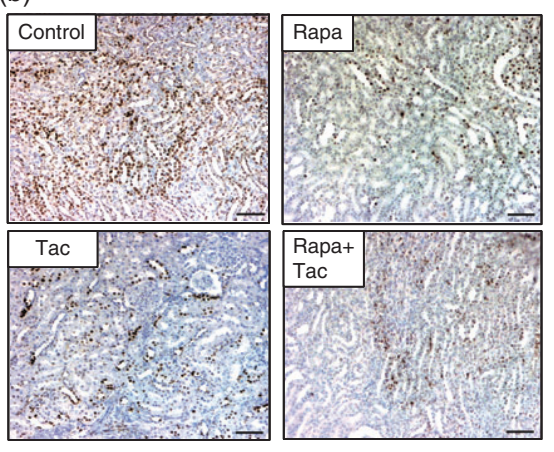

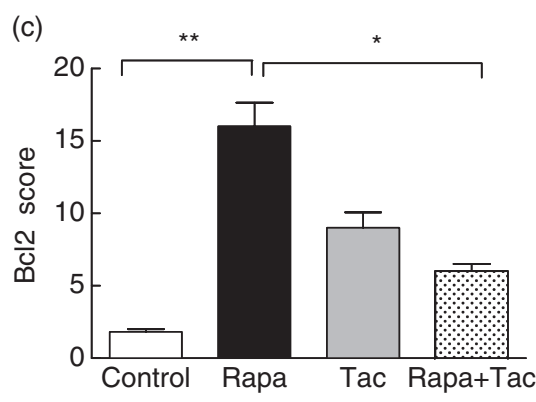

the graft in a syngeneic rat model. The difference observed in our experiments, compared to Lieberthal et al. [40], may result from the administration setting. Once the injury is caused, rapamycin delays ATN recovery but the early administration of rapamycin, i.e. before the injury is caused, may explain the different beneficial effects observed in this exploratory study. Immunosuppressive treatment was administered in a single dose only to donor animals, $12 \mathrm{~h}$ before ablation. Several authors using the transplant model with rapamycin exposure after $\mathrm{I} / \mathrm{R}$ injury support the hypothesis that rapamycin compromises renal function by impairing recovery rather than increasing injury severity $[19,40]$. In particular, Fuller et al. have demonstrated that serum creatinine in rapamycin-treated groups takes longer to recover [42]. These results show coherence regarding the specific impact of rapamycin on injured kidney. The data presented in our exploratory work could provide new evidence for the use of rapamycin as a potent non-nephrotoxic immunosuppressant for its use in donors in the DGF setting.

The exact mechanism underlying the effect described for rapamycin or tacrolimus on renal I/R injury has not been explained completely. The protection by donor preconditioning has been associated with a reduction in the inflammatory response to reperfusion. Accordingly, the proinflammatory cytokines TNF- $\alpha$ and IL- 6 were reduced by donor preconditioning with immunosuppressive treatment drugs. Other studies have also described that rapamycin suppresses IL-6 production, and that this may be associated with regulatory $\mathrm{T}$ cell $\left(\mathrm{T}_{\text {reg }}\right)$ induction and with a decrease in the T helper 17 (Th1) population $[43,44]$. Regarding apoptosis, the improvement observed in the rapamycin group could be explained by 
(a)
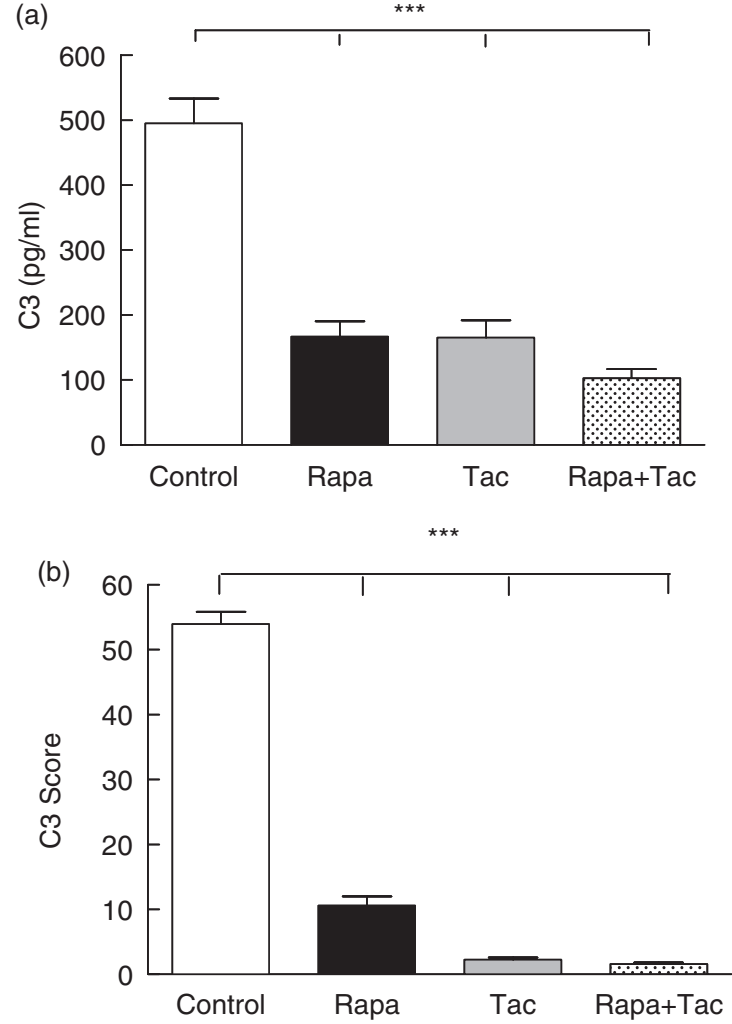

(c)

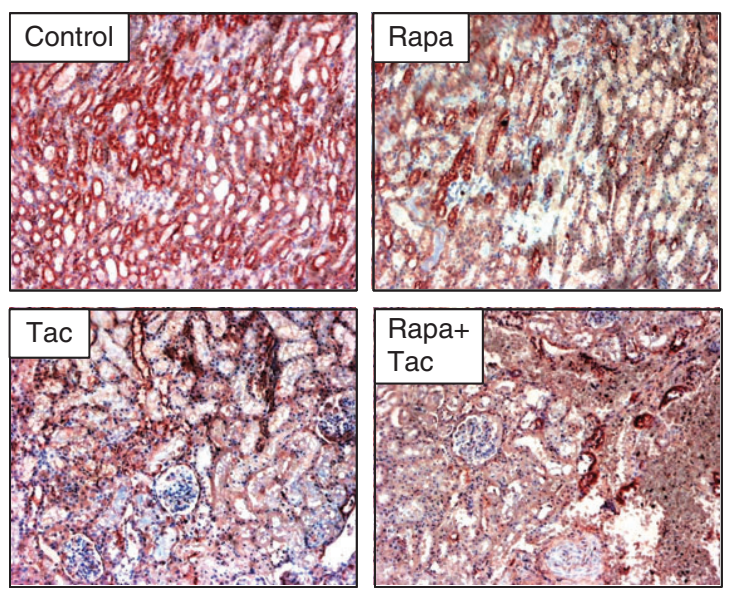

Fig. 4. C3 determination after transplantation. (a) Plasma C3 complement fraction (C3) was measured by radial immunodiffusion. Values are expressed as the difference between post-transplantation minus pretransplantation for each group. (b) Presence of C3 was assessed in 10 consecutive cortex and medulla fields. The extension rather than intensity of these markers was evaluated using a semiquantitative score. (c) Immunohistochemical demonstration of C3 expression in kidney sections from transplanted animals without treatment or treated with rapamycin, tacrolimus or the combination of both $(\mathrm{A} \times 100)$. Values are mean \pm standard deviation $(n=6)$. ${ }^{* *} P<0.001$ for comparison to control group.

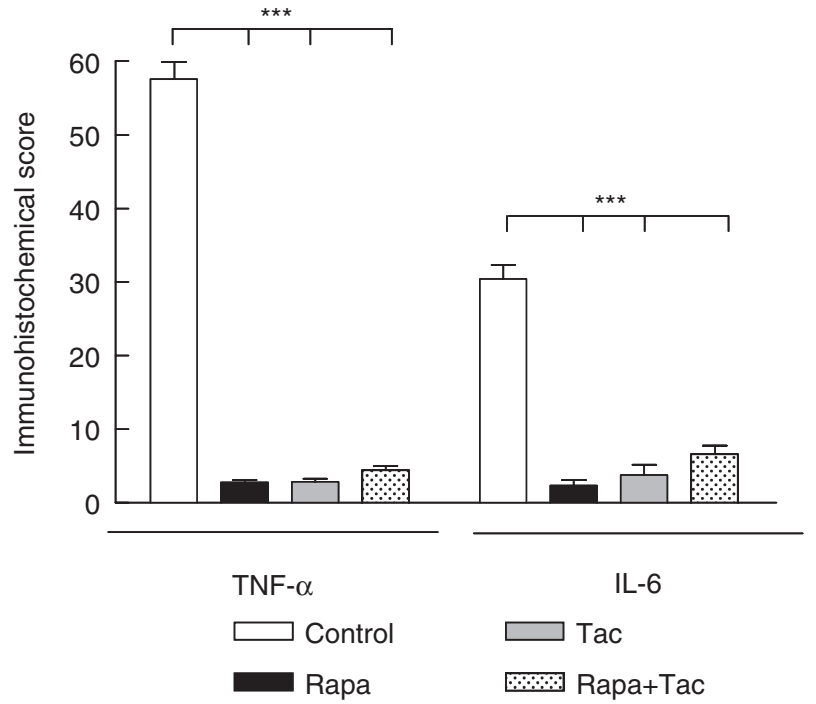

Fig. 5. Score of tumour necrosis factor (TNF)- $\alpha$ and interleukin (IL) -6 after transplantation. The score of TNF- $\alpha$ and IL- 6 were graded on a scale from 1 to 5 by using microscopic criteria as described in Materials and methods. Values are mean \pm standard deviation $(n=6) .{ }^{* *} P<0.001$ for comparison to control group.

in-situ up-regulation of Bcl-2, a specifically anti-apoptotic gene. Remarkably, the increased Bcl-2 expression could not be observed in animals treated with tacrolimus, which also presented more TUNEL-positive cells than those animals treated with rapamycin. However, preconditioning with tacrolimus has a clear anti-apoptotic effect, as it has been shown that tacrolimus diminishes the levels of Fas, Fas-ligand and caspases 1 and 3, which occur with I/R injury [16]. The decrease in apoptosis observed in immunosuppressive treatment groups could be explained partially by the decreased in-situ expression of TNF- $\alpha$, a known inflammatory mediator related to extrinsic pathway of apoptosis inducing apoptosis in renal epithelial cells $[45,46]$. Similarly, the observed decrease in C3 systemic and local levels could be another reason to explain why preconditioning improves clinical outcomes, as a relationship between apoptosis and complement generation in I/R injury is well established $[47,48]$. In a warm ischaemia model, Thurman et al. have shown even higher systemic levels of C3 than in our results, although the measurement was taken in a different time-frame $(8 \mathrm{~h}$ post-I/R injury) [49]. An up-regulated in-situ expression of C3 and caspase 3 can be seen as soon as $2 \mathrm{~h}$ following I/R injury [50]. In our work, with a 3 -h cold ischaemia model, the reduction in plasmatic levels of C3 in immunosuppressive treatment groups could be related to lower expression of C3 observed in situ. Once again, the combined treatment with rapamycin and tacrolimus presented the lowest levels of plasma C3 and local C3 expression.

One of the most important approaches to administer immunosuppressive drugs to the donor begins with the 
study carried out by Farrar et al., showing that C3-deficient kidneys are protected from ischaemic damage after posttransplantation into syngeneic recipient mice with normal serum complement activity; i.e. kidney-derived C3, not serum C3, drives the expression of I/R injury [6]. C3 is synthesized by tubular, mesangial and endothelial cells and contributes to the inflammatory process in kidney transplantation and is up-regulated rapidly after I/R [51]. Complement damaging effects depend mainly on the cleavage of $\mathrm{C} 3$, which is the central component on which all activation pathways converge. This activation may occur via the mannose-binding lectin pathway as well as through the alternative pathway in kidney transplant [52]. C3 cleavage is an essential part of the process ending in the membrane attack complex synthesis which, in turn, could lead to TNF- $\alpha$ and IL- 6 production promoting injury [53]. The mechanism by which both drugs attenuate local and systemic C3 expression is still unknown and needs to be explained.

In our exploratory study, the combination of a calcineurin inhibitor and inhibitors of mTOR diminishes the in-situ generation of proinflammatory mediators; in addition, this combination up-regulates cytoprotective genes. As a result of this process there is diminished necrosis and apoptosis of tubular epithelial cells and, in turn, an improvement in renal clinical function.

Several research groups are studying donor treatment and it may be applied clinically in the near future. However, our experimental model could not be transferred directly to a cadaveric donor transplant model, because brain death of the donor has not been considered. Brain death is a strong proinflammatory event that results in the activation of several pathways [54]. However, we believe that the model could be clinically useful for those patients with living donors who require prolonged bench surgery, or for those patients included in donor pair programmes requiring a longer time of cold ischaemia. As there is no evidence of immunosuppression to donors in living donors, this issue should be debated within a bioethical framework.

To our knowledge, this is one of the few studies showing evidence of a lower I/R injury with combined immunosuppressive treatment of donors using a syngeneic rat model. The use of immunosuppressive drugs administered to donors has attenuated the $\mathrm{I} / \mathrm{R}$ injury process and this was demonstrated by a marked necrosis and apoptosis decrease in renal tubular epithelial cells. Further studies based on this exploratory study would describe the use of immunosuppressive treatment to the donor to improve the quality of the organ to be transplanted.

\section{Acknowledgement}

The authors thank Professor Dr Enrique Portiansky for his assistance in the quantification of optical densities and areas of IHC.

\section{Disclosures}

The authors of this manuscript have no conflicts of interest to disclose.

\section{References}

1 Tilney NL, Guttmann RD. Effects of initial ischemia/reperfusion injury on the transplanted kidney. Transplantation 1997; 64:945-7.

2 Shoskes DA, Halloran PF. Delayed graft function in renal transplantation: etiology, management and long-term significance. J Urol 1996; 155:1831-40.

3 Jang HR, Ko GJ, Wasowska BA, Rabb H. The interaction between ischemia-reperfusion and immune responses in the kidney. J Mol Med 2009; 87:859-64.

4 Thurman JM. Triggers of inflammation after renal ischemia/ reperfusion. Clin Immunol 2007; 123:7-13.

5 Donnahoo KK, Shames BD, Harken AH, Meldrum DR. Review article: the role of tumor necrosis factor in renal ischemiareperfusion injury. J Urol 1999; 162:196-203.

6 Farrar CA, Zhou W, Lin T, Sacks SH. Local extravascular pool of C3 is a determinant of postischemic acute renal failure. FASEB J 2006; 20:217-26.

7 Genesca M, Sola A, Hotter G. Actin cytoskeleton derangement induces apoptosis in renal ischemia/reperfusion. Apoptosis 2006; 11:563-71.

8 Yamada K, Miwa T, Liu J, Nangaku M, Song WC. Critical protection from renal ischemia reperfusion injury by CD55 and CD59. J Immunol 2004; 172:3869-75.

9 Zhou W, Farrar CA, Abe K et al. Predominant role for C5b-9 in renal ischemia/reperfusion injury. J Clin Invest 2000; 105:1363-71.

10 Vakeva AP, Agah A, Rollins SA, Matis LA, Li L, Stahl GL. Myocardial infarction and apoptosis after myocardial ischemia and reperfusion: role of the terminal complement components and inhibition by anti-C5 therapy. Circulation 1998; 97:2259-67.

11 Boros P, Bromberg JS. New cellular and molecular immune pathways in ischemia/reperfusion injury. Am J Transplant 2006; 6:652-8.

12 Kuecuek O, Mantouvalou L, Klemz R et al. Significant reduction of proinflammatory cytokines by treatment of the brain-dead donor. Transplant Proc 2005; 37:387-8.

13 Kotsch K, Ulrich F, Reutzel-Selke A et al. Methylprednisolone therapy in deceased donors reduces inflammation in the donor liver and improves outcome after liver transplantation: a prospective randomized controlled trial. Ann Surg 2008; 248:1042-50.

14 Gottmann U, Brinkkoetter PT, Hoeger S et al. Atorvastatin donor pretreatment prevents ischemia/reperfusion injury in renal transplantation in rats: possible role for aldose-reductase inhibition. Transplantation 2007; 84:755-62.

15 Goncalves GM, Cenedeze MA, Feitoza CQ et al. The role of immunosuppressive drugs in aggravating renal ischemia and reperfusion injury. Transplant Proc 2007; 39:417-20.

16 Yang CW, Ahn HJ, Han HJ et al. Pharmacological preconditioning with low-dose cyclosporine or FK506 reduces subsequent ischemia/reperfusion injury in rat kidney. Transplantation 2001; 72:1753-9.

17 Cicora F, Lausada N, Vasquez DN et al. Protective effect of immunosuppressive treatment before orthotopic kidney autotransplantation. Transplant Immunol 2011; 24:107-12. 
18 Coombes JD, Mreich E, Liddle C, Rangan GK. Rapamycin worsens renal function and intratubular cast formation in protein overload nephropathy. Kidney Int 2005; 68:2599-607.

19 Andoh TF, Burdmann EA, Fransechini N, Houghton DC, Bennett WM. Comparison of acute rapamycin nephrotoxicity with cyclosporine and FK506. Kidney Int 1996; 50:1110-17.

20 Lieberthal W, Fuhro R, Andry CC et al. Rapamycin impairs recovery from acute renal failure: role of cell-cycle arrest and apoptosis of tubular cells. Am J Physiol Renal Physiol 2001; 281:F693-706.

21 Puglisi RN, Strande L, Santos M, Schulte G, Hewitt CW, Whalen TV. Beneficial effects of cyclosporine and rapamycin in small bowel ischemic injury. J Surg Res 1996; 65:115-18.

22 Inman SR, Davis NA, Olson KM, Lukaszek VA, McKinley MR, Seminerio JL. Rapamycin preserves renal function compared with cyclosporine A after ischemia/reperfusion injury. Urology 2003; 62:750-4.

23 Lui SL, Chan KW, Tsang R, Yung S, Lai KN, Chan TM. Effect of rapamycin on renal ischemia-reperfusion injury in mice. Transplant Int 2006; 19:834-9.

24 Khan S, Salloum F, Das A, Xi L, Vetrovec GW, Kukreja RC. Rapamycin confers preconditioning-like protection against ischemiareperfusion injury in isolated mouse heart and cardiomyocytes. J Mol Cell Cardiol 2006; 41:256-64.

25 Frantz B, Nordby EC, Bren G et al. Calcineurin acts in synergy with PMA to inactivate I kappa B/MAD3, an inhibitor of NF-kappa B. EMBO J 1994; 13:861-70.

26 Sakr MF, Hassanein TI, Zetti GM, Van Thiel DH. FK 506 ameliorates the hepatic injury associated with ischemia. Life Sci 1990; 47:687-91.

27 Sakr M, Zetti G, McClain C et al. The protective effect of FK506 pretreatment against renal ischemia/reperfusion injury in rats. Transplantation 1992; 53:987-91.

28 Kouwenhoven EA, de Bruin RW, Bajema IM, Marquet RL, Ijzermans JN. Cold ischemia augments allogeneic-mediated injury in rat kidney allografts. Kidney Int 2001; 59:1142-8.

29 Kaminska D, Tyran B, Mazanowska O et al. Cytokine gene expression in kidney allograft donor biopsies after cold ischemia and reperfusion using in situ RT-PCR analysis. Transplant Proc 2003; 35:2155-6.

30 de Vries DK, Lindeman JH, Ringers J, Reinders ME, Rabelink TJ, Schaapherder AF. Donor brain death predisposes human kidney grafts to a proinflammatory reaction after transplantation. Am J Transplant 2011; 11:1064-70.

31 Guan X, Dei-Anane G, Liang R et al. Donor preconditioning with taurine protects kidney grafts from injury after experimental transplantation. J Surg Res 2008; 146:127-34.

32 Huang H, He Z, Roberts LJ 2nd, Salahudeen AK. Deferoxamine reduces cold-ischemic renal injury in a syngeneic kidney transplant model. Am J Transplant 2003; 3:1531-7.

33 Wells WA, Rainer RO, Memoli VA. Equipment, standardization, and applications of image processing. Am J Clin Pathol 1993; 99:48-56.

34 Shihab FS, Bennett WM, Andoh TF. Donor preconditioning with a calcineurin inhibitor improves outcome in rat syngeneic kidney transplantation. Transplantation 2009; 87:326-9.

35 Smith KD, Wrenshall LE, Nicosia RF et al. Delayed graft function and cast nephropathy associated with tacrolimus plus rapamycin use. J Am Soc Nephrol 2003; 14:1037-45.

36 McTaggart RA, Gottlieb D, Brooks J et al. Sirolimus prolongs recovery from delayed graft function after cadaveric renal transplantation. Am J Transplant 2003; 3:416-23.
37 Novick AC, Hwei HH, Steinmuller D et al. Detrimental effect of cyclosporine on initial function of cadaver renal allografts following extended preservation. Results of a randomized prospective study. Transplantation 1986; 42:154-8.

38 Ninova D, Covarrubias M, Rea DJ, Park WD, Grande JP, Stegall MD. Acute nephrotoxicity of tacrolimus and sirolimus in renal isografts: differential intragraft expression of transforming growth factor-betal and alpha-smooth muscle actin. Transplantation 2004; 78:338-44.

39 Abraham RT. Mammalian target of rapamycin: immunosuppressive drugs uncover a novel pathway of cytokine receptor signaling. Curr Opin Immunol 1998; 10:330-6.

40 Lieberthal W, Fuhro R, Andry C, Patel V, Levine JS. Rapamycin delays but does not prevent recovery from acute renal failure: role of acquired tubular resistance. Transplantation 2006; 82: $17-22$.

41 Podder H, Stepkowski SM, Napoli K, Kahan BD. Pharmacokinetic interactions between sirolimus and cyclosporine exacerbate renal dysfunction. Transplant Proc 2001; 33:2-29.

42 Fuller TF, Freise CE, Serkova N, Niemann CU, Olson JL, Feng S. Sirolimus delays recovery of rat kidney transplants after ischemiareperfusion injury. Transplantation 2003; 76:1594-9.

43 Sun Q, Liu Q, Zheng Y, Cao X. Rapamycin suppresses TLR4triggered IL-6 and PGE(2) production of colon cancer cells by inhibiting TLR4 expression and NF-kappaB activation. Mol Immunol 2008; 45:2929-36.

44 Kopf H, de la Rosa GM, Howard OM, Chen X. Rapamycin inhibits differentiation of Th17 cells and promotes generation of FoxP3+ T regulatory cells. Int Immunopharmacol 2007; 7:1819-24.

45 Cunningham PN, Dyanov HM, Park P, Wang J, Newell KA, Quigg RJ. Acute renal failure in endotoxemia is caused by TNF acting directly on TNF receptor-1 in kidney. J Immunol 2002; 168:581723.

46 Misseri R, Meldrum DR, Dinarello CA et al. TNF-alpha mediates obstruction-induced renal tubular cell apoptosis and proapoptotic signaling. Am J Physiol Renal Physiol 2005; 288:F406-11.

47 Zheng X, Feng B, Chen G et al. Preventing renal ischemiareperfusion injury using small interfering RNA by targeting complement 3 gene. Am J Transplant 2006; 6:2099-108.

48 Siegel RM. Caspases at the crossroads of immune-cell life and death. Nat Rev Immunol 2006; 6:308-17.

49 Thurman JM, Ljubanovic D, Royer PA et al. Altered renal tubular expression of the complement inhibitor Crry permits complement activation after ischemia/reperfusion. J Clin Invest 2006; 116: 357-68.

50 Sacks SH, Zhou W. Locally produced complement and its role in renal allograft rejection. Am J Transplant 2003; 3:927-32.

51 Morgan BP, Gasque P. Extrahepatic complement biosynthesis: where, when and why? Clin Exp Immunol 1997; 107:1-7.

52 de Vries B, Walter SJ, Peutz-Kootstra CJ, Wolfs TG, van Heurn LW, Buurman WA. The mannose-binding lectin-pathway is involved in complement activation in the course of renal ischemia-reperfusion injury. Am J Pathol 2004; 165:1677-88.

53 David S, Biancone L, Caserta C, Bussolati B, Cambi V, Camussi G. Alternative pathway complement activation induces proinflammatory activity in human proximal tubular epithelial cells. Nephrol Dial Transplant 1997; 12:51-6.

54 Pratschke J, Wilhelm MJ, Kusaka M et al. Accelerated rejection of renal allografts from brain-dead donors. Ann Surg 2000; 232: $263-71$. 